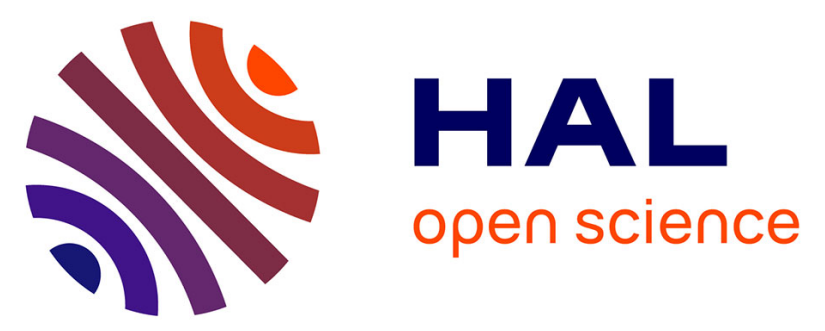

\title{
Cross-term Splittings Due to the Orientational Inequivalence of Proton Magnetic Shielding Tensors: Do Water Molecules Trapped in Crystals Hop or Tunnel?
}

Diego Carnevale, Philippe Pelupessy, Geoffrey Bodenhausen

\section{- To cite this version:}

Diego Carnevale, Philippe Pelupessy, Geoffrey Bodenhausen. Cross-term Splittings Due to the Orientational Inequivalence of Proton Magnetic Shielding Tensors: Do Water Molecules Trapped in Crystals Hop or Tunnel?. Journal of Physical Chemistry Letters, 2019, 10 (10), pp.3224-3231. 10.1021/acs.jpclett.9b00914 . hal-02285924

\section{HAL Id: hal-02285924 \\ https: / hal.sorbonne-universite.fr/hal-02285924}

Submitted on 18 Sep 2019

HAL is a multi-disciplinary open access archive for the deposit and dissemination of scientific research documents, whether they are published or not. The documents may come from teaching and research institutions in France or abroad, or from public or private research centers.
L'archive ouverte pluridisciplinaire HAL, est destinée au dépôt et à la diffusion de documents scientifiques de niveau recherche, publiés ou non, émanant des établissements d'enseignement et de recherche français ou étrangers, des laboratoires publics ou privés. 
Cross-term splittings due to the orientational inequivalence of proton magnetic shielding tensors: Do water molecules trapped in crystals hop or tunnel?

\title{
Cross-term splittings due to the orientational inequivalence of proton magnetic shielding tensors: Do water molecules trapped in crystals hop or tunnel?
}

\author{
Diego Carnevale,* Philippe Pelupessy and Geoffrey Bodenhausen
}

Laboratoire des biomolécules, LBM, Département de chimie, École normale supérieure, PSL University, Sorbonne Université, CNRS, 75005 Paris, France

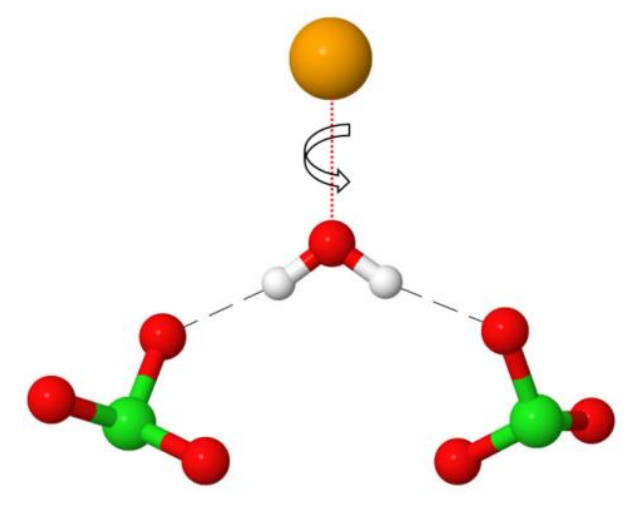

The Journal of Physical Chemistry Letters (Accepted May $6^{\text {th }}$ 2019)

http://dx.doi.org/10.1021/acs.jpclett.9b00914

Corresponding author: Diego Carnevale

diego.carnevale@ens.fr 


\begin{abstract}
Water molecules trapped in crystals of barium chlorate monohydrate have been investigated by magic angle spinning proton NMR spectroscopy in the temperature range 110$300 \mathrm{~K}$. At high temperatures, a single spinning sideband pattern is observed. Below $150 \mathrm{~K}$ however, two interleaved spinning sideband manifolds appear, with distinct centerbands that do not coincide with the average isotropic chemical shift seen at high temperatures. This hitherto unknown "cross-term splitting" results from the interplay of the homonuclear dipoledipole coupling and two anisotropic proton shielding tensors that have identical principal components but non-equivalent orientations. The resulting cross terms cannot be averaged out by rotation about the magic angle. The analysis of the exchange-induced broadening, coalescence and narrowing of the cross-term splitting in MAS spectra allows one to estimate the rate of exchange of the two protons between 140 and $190 \mathrm{~K}$. The experimental data is compared with ${ }^{2} \mathrm{H}$ and ${ }^{1} \mathrm{H}$ NMR studies of the same sample reported in the literature. DFT methods are utilized to estimate the thermal activation energy for a two-fold hopping process of proton exchange about the $\mathrm{H}-\mathrm{O}-\mathrm{H}$ bisector. The Bell-Limbach model allows one to take into account contributions due to incoherent quantum tunneling in the low temperature regime.
\end{abstract}

\title{
Key words
}

Orientational Inequivalence, Quantum Tunneling, Magic-Angle Spinning, Solid-State NMR, Anisotropic Motional Dynamics, Chemical Exchange, Homonuclear Dipolar Interaction, Chemical Shift Anisotropy, Cross-Term Splitting. 


\section{Introduction}

Despite its deceptive simplicity, $\mathrm{H}_{2} \mathrm{O}$ shows a variety of surprising properties, whether in bulk liquids or solids, confined in nanocavities, or near the surface of macromolecules such as proteins or nucleic acids. ${ }^{1-7}$ To date, there is no established general consensus on the structural organization of $\mathrm{H}_{2} \mathrm{O}$ molecules on the atomic scale. Hydrogen bonds and dynamic processes such as proton exchange further complicate the scenarios. Quantum effects have been considered to shed light on this molecular jumble. ${ }^{8-10}$ Recently, quantum tunneling dynamics have been reported for water molecules confined in nanocavities of beryl crystals ${ }^{11,12}$ and in gasphase clusters like $\left(\mathrm{H}_{2} \mathrm{O}\right)_{6}{ }^{13}$

The chemical shift anisotropy (CSA) of protons in water molecules is of particular interest since it has the potential of lifting the magnetic equivalence of the two protons. Inter alia, this may give access to para-water, i.e., by inducing an excess or a deficiency of the population of the singlet state $\left|S_{0}\right\rangle=1 / \sqrt{2}[|\alpha \beta\rangle-|\beta \alpha\rangle]$ with respect to the average population of the triplet states $\left|T_{+1}\right\rangle=|\alpha \alpha\rangle,\left|T_{-1}\right\rangle=|\beta \beta\rangle$ and $\left|T_{0}\right\rangle=1 / \sqrt{2}[|\alpha \beta\rangle+|\beta \alpha\rangle]$, either by going to very low spin temperatures, ${ }^{14,15}$ or by suitable manipulations with radio-frequency ( $r$ ) pulses. ${ }^{16,17}$

In the absence of motional averaging, the shielding tensors of the two protons in a water molecule have different orientations with respect to one another. Except if the crystal environment breaks the symmetry of the $\mathrm{H}_{2} \mathrm{O}$ molecules (which is not the case in our study), the two protons are chemically equivalent, i.e., have identical tensors in their principal axis systems, with identical isotropic shifts $\delta_{\text {iso, }}$ identical anisotropies $\Delta_{c s}$ and identical asymmetry parameters $\eta$ cs. When immersed in a magnetic field however, the difference of the orientations of the two shielding tensors renders the two protons inequivalent, except for some specific orientations. More specifically, although with identical isotropic shifts $\delta_{\text {iso }}$ and identical rank-2 anisotropic shift tensors $\Delta_{C S} \times\left\{-\eta_{C S} / \sqrt{6}, 0, \quad 1,0,-\eta_{C S} / \sqrt{6}\right\}$, the Euler angles relating the two interactions when they are expressed in a common crystal frame $C$ are different for spins $I$ and $S$, i.e., $\Omega_{P C}^{(I)}=\left(\begin{array}{lll}0, & 0, & 0\end{array}\right) \neq \Omega_{P C}^{(S)}=\left(\begin{array}{lll}\alpha_{P C}^{(S)}, & \beta_{P C}^{(S)}, & \gamma_{P C}^{(S)}\end{array}\right)$, where the principal axis frame $P$ of spin $I$ is 
Cross-term splittings due to the orientational inequivalence of proton magnetic shielding tensors: Do water molecules trapped in crystals hop or tunnel?

arbitrarily chosen as common frame $C$. We refer to this type of non-equivalence as orientational inequivalence.

We have investigated the protons of water molecules trapped in barium chlorate monohydrate, $\mathrm{Ba}\left(\mathrm{ClO}_{3}\right)_{2} \cdot \mathrm{H}_{2} \mathrm{O}$, by means of variable temperature magic-angle spinning (MAS) proton NMR spectroscopy over a temperature range $110<T<300 \mathrm{~K}$. This sample bears analogies to hydrated gypsum $\mathrm{CaSO}_{4} \cdot 2 \mathrm{H}_{2} \mathrm{O}$ that features characteristic proton NMR spectra that were first described by Pake. ${ }^{18}$ Similar dynamic processes have been studied in $\mathrm{Ba}\left(\mathrm{ClO}_{3}\right)_{2} \cdot \mathrm{D}_{2} \mathrm{O}$ by Long et al. ${ }^{19}$ by means of deuterium NMR spectroscopy, albeit over a narrower temperature range $154<T<291 \mathrm{~K}$. We have independently simulated the deuterium lineshapes observed by Long et al. ${ }^{19}$ and found good agreement with their exchange rates. Proton and oxygen-17 NMR investigations of the same sample have also been reported. ${ }^{20,21}$ In this work, we show numerical simulations of proton spectra over an extended temperature range $110<T<300 \mathrm{~K}$ that take into account the presence of exchange and agree remarkably well with the unusual sideband patterns of our experimental MAS spectra. The experimental evidence is compared with previous ${ }^{2} \mathrm{H}$ and ${ }^{1} \mathrm{H}$ NMR studies. Within experimental errors over the $140<\mathrm{T}<190 \mathrm{~K}$ temperature range, we find an activation energy which is higher than that reported in the literature. This finding is corroborated by density functional theory (DFT) calculations of the thermal activation energy for the two-fold flip about the $\mathrm{H}-\mathrm{O}-\mathrm{H}$ bisector. Deviations from linearity in the Arrhenius plot are rationalized by means of the Bell-Limbach model ${ }^{22,23}$ that takes into account incoherent quantum tunneling contributions at low temperatures. The unusual spinning sideband patterns observed at low temperatures result from CSA-dipole cross terms that lead to fourth-rank interactions that cannot be eliminated by spinning about the magic angle. ${ }^{24,25}$

\section{Results and discussion}

The crystal structure of $\mathrm{Ba}\left(\mathrm{ClO}_{3}\right)_{2} \cdot \mathrm{H}_{2} \mathrm{O}$ is shown in Figure 1 . The parameters of the monoclinic unit cell are $a=8.92 \AA, b=7.83 \AA$ and $c=9.43 \AA$, with $\beta=93.39^{\circ}$ and a space group $C 2 / c{ }^{26}$ Four water molecules (shown in cyan) are found in each unit cell. Each water molecule is 


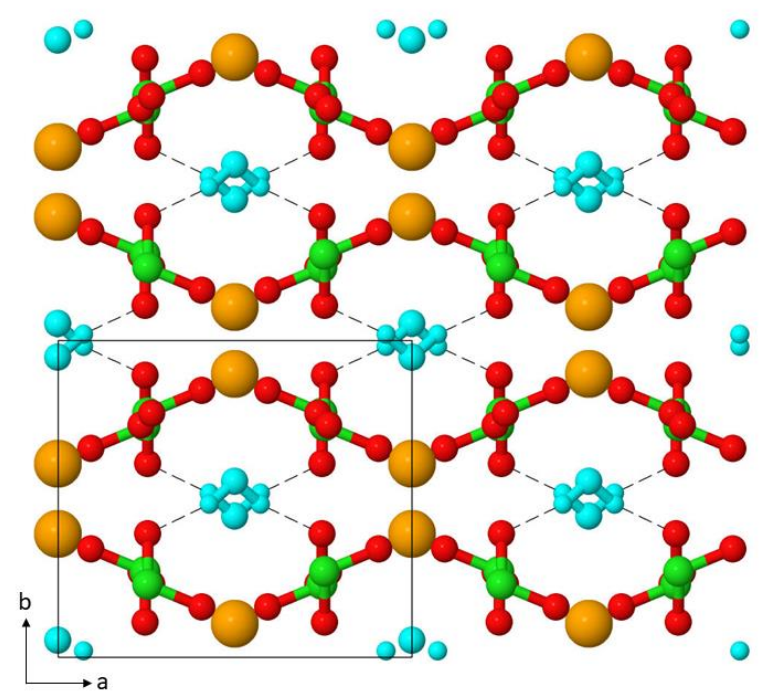

Figure 1 Crystal structure of $\mathrm{Ba}\left(\mathrm{ClO}_{3}\right)_{2} \cdot \mathrm{H}_{2} \mathrm{O}$, viewed down the $c$-axis. Water molecules are shown in cyan whereas all remaining barium, chlorine, and oxygen atoms are shown in orange, green and red, respectively. Water molecules are stacked along channels running down the $c$-axis, with alternating orientations in subsequent planes. Hydrogen bonds between water protons and oxygen atoms of chlorate anions are indicated by dashed lines. The lattice parameters obtained by neutron diffraction agree with the structure optimized with planewave-pseudopotential DFT methods, yielding $a=9.078, b=$ 8.061 and $c=9.591 \AA$, with $\beta=92.698^{\circ}$. The intramolecular ${ }^{1} \mathrm{H}-{ }^{1} \mathrm{H}$ dipolar coupling is ca. $30 \mathrm{kHz}$, whereas intermolecular couplings between neighboring $\mathrm{H}_{2} \mathrm{O}$ molecules are smaller than ca. $2 \mathrm{kHz}$.

involved in two hydrogen bonds of length $1.92 \AA$ with oxygen atoms of two different chlorate anions. The oxygen atom of each water molecule is coordinated with one $\mathrm{Ba}^{2+}$ cation, at a distance of $2.74 \AA$, along a vector parallel to the $b$-axis. This structure defines channels of ca. 4.5 $\AA$ across that run parallel to the $c$-axis, where water molecules are found at intervals of $4.88 \AA$, with a $180^{\circ}$ alternation of the orientations of the $\mathrm{H}-\mathrm{O}-\mathrm{H}$ bisecting vectors. Intermolecular dipolar couplings between the closest protons $(<2 \mathrm{kHz})$ are therefore much smaller than intramolecular couplings (ca. $30 \mathrm{kHz}$ ), so that each water molecule may be considered to be reasonably isolated. This is consistent with the quasi-ideal Pake patterns observed in static proton NMR spectra of this sample. ${ }^{27,28}$ These patterns are compatible with the fact that the dominant intramolecular dipolar interactions is not affected by the swapping of the protons through a two-fold flip about the $\mathrm{H}-\mathrm{O}-\mathrm{H}$ bisector or through tunneling. Therefore, the intramolecular dipolar Hamiltonian is an invariant of motion in our case. ${ }^{16,17}$ On the other hand, fast swapping of the protons results in a partial averaging of the two shielding tensors, resulting in two collinear tensors, thus removing their orientational inequivalence above $200 \mathrm{~K}$. In this 
case, we can speak of a quasi-invariant of motion for the CSA Hamiltonian. We have previously investigated the CSA tensors of the protons of trapped water molecules by solid-state NMR under both static and magic-angle spinning conditions at $300 \mathrm{~K} \cdot{ }^{27}$ The values measured at room temperature agree remarkably well with those calculated by means of DFT methods for a periodic lattice when fast $180^{\circ}$ flips about the $\mathrm{H}-\mathrm{O}-\mathrm{H}$ bisectors are assumed to occur rapidly. In the absence of motional averaging, these calculations show that the two protons have shielding tensors that are identical in size and shape. ${ }^{27}$ In this study, we have repeated these DFT calculations at a higher level of theory. The resulting NMR parameters are $\Delta_{C S}=-15.4 \mathrm{ppm}$, $\eta_{C S}=0.1$, with Euler angles relating the two shielding tensors $\left(90.13^{\circ}, 65.37^{\circ}, 90.12^{\circ}\right)$, in good agreement with our previous study. ${ }^{27}$ The angle $\beta=65.37^{\circ}$ between the two main components $\delta_{z z}$ of the shielding tensors is compatible with the tetrahedral angle, i.e., $\beta^{\prime}=(180-65.37)^{\circ}=$ $114.63^{\circ}$. Figure 2 shows numerical simulations of proton spectra assuming these latter

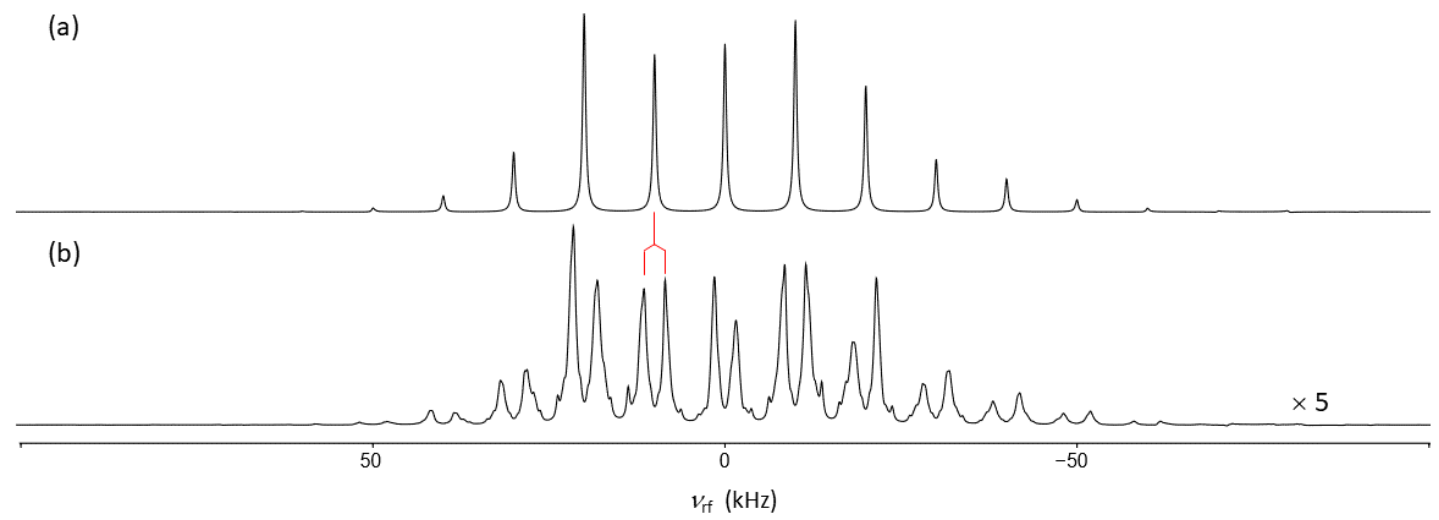

Figure 2 Numerical simulations of proton NMR spectra of an isolated $\mathrm{H}_{2} \mathrm{O}$ molecule in a polycrystalline $\mathrm{Ba}\left(\mathrm{ClO}_{3}\right)_{2} \cdot \mathrm{H}_{2} \mathrm{O}$ powder spinning at a rate $v_{R}=10 \mathrm{kHz}$ in a field $B_{0}=18.8 \mathrm{~T}(800 \mathrm{MHz})$, using intramolecular ${ }^{1} \mathrm{H}^{-1} \mathrm{H}$ dipole-dipole and ${ }^{1} \mathrm{H}$ shielding tensors calculated by DFT. (a) Spectrum simulated assuming that the two proton shielding tensors are both orthogonal to the dipolar tensor and collinear with respect to each other, as expected when the CSA tensors are averaged by rapid exchange at high temperatures. (b) Spectrum that is expected at low temperatures where the dynamics are frozen, so that the CSA tensors of the two protons of each water molecule become "orientationally inequivalent", i.e., no longer collinear, although they retain the same principal components. One of the hitherto unknown "cross-term splittings" of ca. $3 \mathrm{kHz}$ between the two sideband manifolds is emphasized by a tree diagram in red. 
Cross-term splittings due to the orientational inequivalence of proton magnetic shielding tensors: Do water molecules trapped in crystals hop or tunnel?

parameters when spinning about the magic angle with $v_{\text {rot }}=10 \mathrm{kHz}$ in a field $B_{0}=18.8 \mathrm{~T}$. The full set of parameters of shielding and dipolar interactions and the relevant sets of Euler angles are given in Supporting Information. Figure 2(a) is based on averaged CSA values $\bar{\Delta}_{C S}=-7.6 \mathrm{ppm}$ and $\bar{\eta}_{C S}=1.0$ (corresponding to proton exchange that is much faster than the differences of the CSA tensors), while Fig. 2(b) shows the spectrum in the absence of motions, as observed at low temperatures. The latter simulation shows how the spinning sideband pattern splits into two interleaved manifolds when exchange is slow. The separation, henceforth called "cross-term splitting", is ca. $3 \mathrm{kHz}$, and is indicated by a red tree diagram for a pair of spinning sidebands. It is worth noting that the lines of the spectrum of Fig. 2(b) are not simple Lorentzians but retain some inhomogeneous features that are typical of forth-rank interactions, similar to secondorder quadrupolar broadenings for spins $I>1 / 2$. This shows the effects of orientational shielding inequivalence on NMR lineshapes.

In order to provide experimental evidence for these observations, proton NMR spectra of a sample of polycrystalline $\mathrm{Ba}\left(\mathrm{ClO}_{3}\right)_{2} \cdot \mathrm{H}_{2} \mathrm{O}$ spinning about the magic angle at $10 \mathrm{kHz}$ were acquired over a range of temperatures $110 \leq T \leq 300 \mathrm{~K}$ in a static field $B_{0}=18.8 \mathrm{~T}$ ( $800 \mathrm{MHz}$ ) (left panel of Fig. 3). As the temperature is decreased, one clearly observes a progressive increase in the width of the lines of the individual sidebands, until a coalescence point in the range $150<T$ $<160 \mathrm{~K}$. Below this temperature, two interleaved sideband manifolds are observed with a splitting of ca. $3 \mathrm{kHz}$, in very good agreement with the simulations of Fig. 2. Simulated spectra assuming DFT parameters for different exchange rates $k_{e x}$ are shown in the right panel of Figure 3. These were calculated in Liouville space, as done routinely to describe exchange phenomena in solution-state NMR. A line broadening of $900 \mathrm{~Hz}$ was applied to all simulated spectra in order to match the full width at half height (FWHH) observed experimentally at high temperatures. It is worth noting that the experimental lines at high temperatures are not purely Lorentzian, in contrast to the lineshapes obtained in silico. This discrepancy may be ascribed to $B_{0}$ inhomogeneities and some degree of disorder in the physical sample. These simulations show a remarkable agreement with the experimental low temperature lineshapes. The exchange rates $k_{e x}$ were chosen to obtain the best resemblance with the experimental spectra and by minimizing differences in linewidths. We ascribe subtle discrepancies between relative 


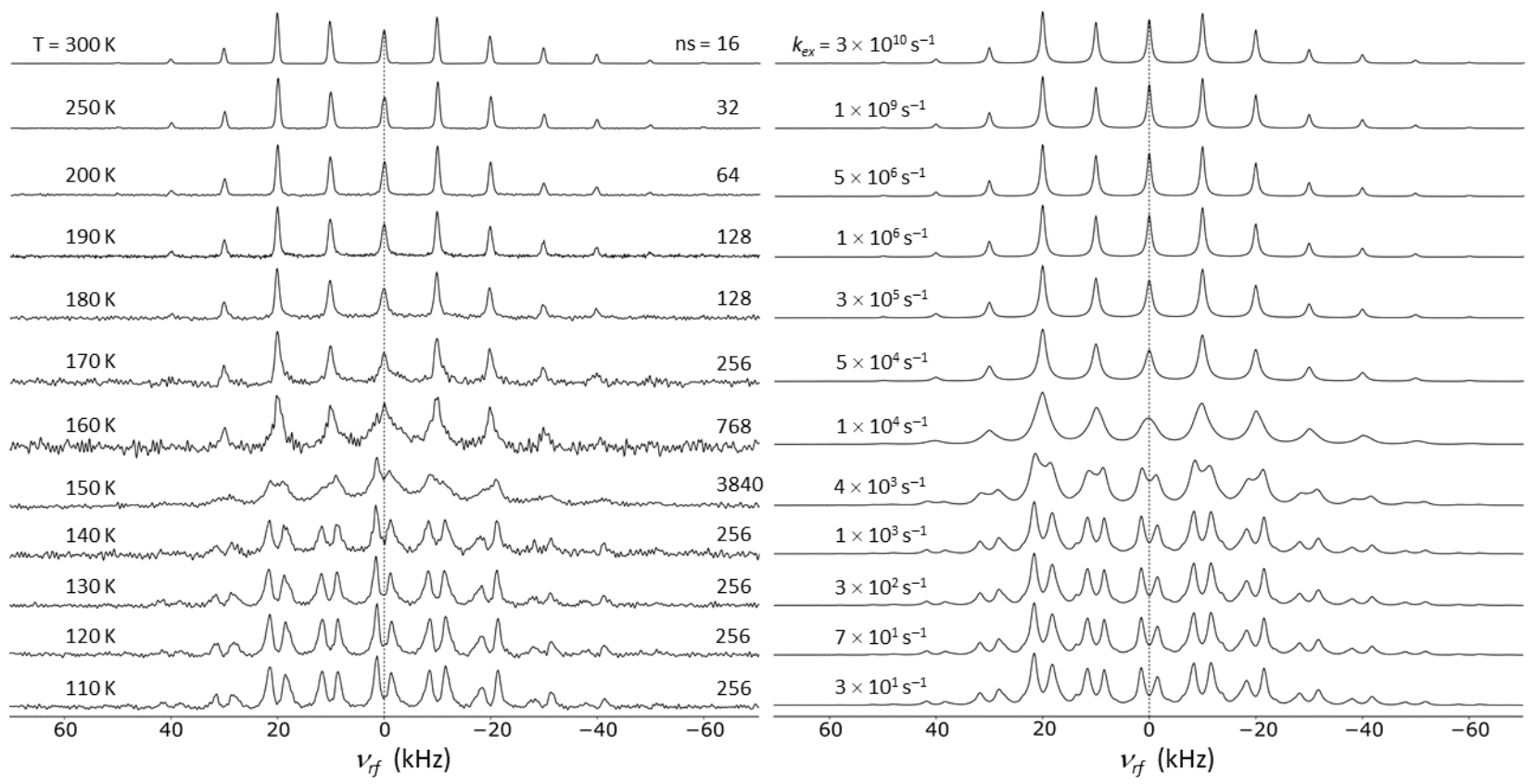

Figure 3 (Left) Experimental proton NMR spectra of polycrystalline $\mathrm{Ba}\left(\mathrm{ClO}_{3}\right)_{2} \cdot \mathrm{H}_{2} \mathrm{O}$ acquired with a rotor-synchronized solid echo $\left(\tau_{\mathrm{R}}=100 \mu \mathrm{s}\right)$ while spinning about the magic angle at $\mathrm{v}_{\mathrm{R}}=10 \mathrm{kHz}$ and $B_{0}=$ 18.8 $\mathrm{T}(800 \mathrm{MHz})$ over the temperature range $110 \leq T \leq 300 \mathrm{~K}$. A recovery delay of $15 \mathrm{~s}$ between subsequent scans was used in all cases. (Right) Numerical simulations of spinning sideband patterns for water molecules with an intramolecular ${ }^{1} \mathrm{H}-{ }^{1} \mathrm{H}$ dipole-dipole interaction of $30 \mathrm{kHz}$ and two orientationally inequivalent CSA tensors that are not collinear although they have the same principal components, with an isotropic chemical shift $\delta_{\text {iso }}=0 \mathrm{ppm}$, an anisotropy $\Delta_{\mathrm{cs}}=-15.4 \mathrm{ppm}$, and an asymmetry $\eta_{c s}=0.12$. The Euler angles relating the various interactions used in the simulations are given in Supporting Materials. The simulations were conducted in the $8 \times 8$ single-quantum Liouville subspace spanned by the basis $\left\{I_{x}\right.$, $\left.I_{y}, S_{x}, S_{y}, 2 I_{x} S_{z}, 2 I_{y} S_{z}, 2 I_{z} S_{x}, 2 I_{z} S_{y}\right\}$ relevant for the chemical shift and dipolar interactions acting on an initial state $I_{x}+S_{x}$. All simulated spectra result from powder averaging over $10 \times 20 \times 10$ crystallite orientations. A Lorentzian line broadening of $900 \mathrm{~Hz}$ was applied to all calculated spectra.

intensities of spinning sidebands in the experimental and simulated spectra to the fact that $T_{1}$ depends on the orientation of the crystallites and is therefore inhomogeneous across the spinning sideband manifolds. ${ }^{19,29}$

Figure 4 shows the experimental and simulated linewidths (black and red data points) extracted from the lineshapes of Fig. 3 vs 1/T. The overall agreement is very satisfying. The coalescence region, characterized by a marked increase in linewidths, is clearly identified. A 900 $\mathrm{Hz}$ line broadening allows one to match the FWHH at high temperatures. However, a mismatch 

water molecules trapped in crystals hop or tunnel?

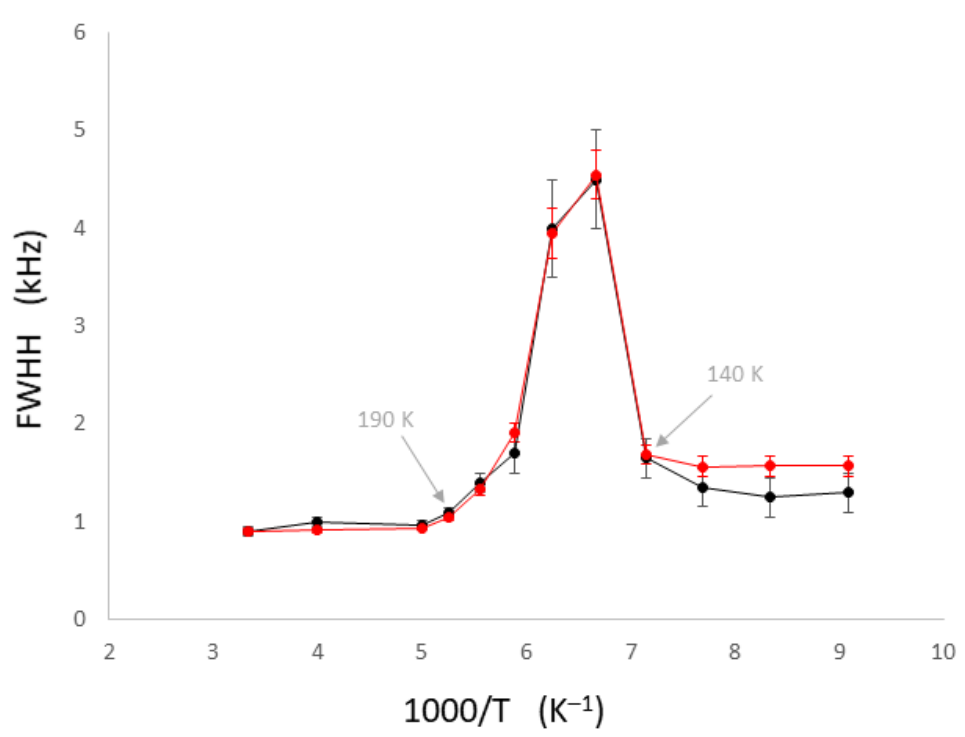

Figure 4 Full widths at half height (FWHH) of the experimental MAS spectra of the left panel of Fig. 3 (black data points). Analogous FWHH of the simulations of the right panel of Fig. 3 (red data). Values were calculated with respect to the centerband. For $\mathrm{T}<140$, the average FWHH for the two lines of the 'doublet' is reported.

in linewidths is observed in the low-temperature region. We ascribe this discrepancy to errors in the Euler angles calculated by DFT methods, despite the improved accuracy of recent calculations. Indeed, a variation of these Euler angles leads to a fine structure (which is perceived as broadening) of each component of the low-temperature doublets (see Supporting Information, Fig. S1). It is also worth noting that the lack of temperature dependence of the linewidths in the first three and last three experimental and simulated spectra indicate that variations $k_{e x}$ rates do not affect the linewidths in these ranges. This is in agreement with the observation that the three spectra in the high-temperature range $200<T<300 \mathrm{~K}$ of Fig. 3 do not show any significant changes in lineshape. Similarly, the experimental proton spectra show little variation in the low-temperature range $110<T<130 \mathrm{~K}$.

Figure 5(a) shows an Arrhenius plot $\ln \left(k_{e x}(T)\right)$ extracted from a comparison of experiments and simulations of Fig. 3 in the critical range $140<T<190 \mathrm{~K}$ represented by black dots. The error bar for the $i^{\text {th }}$ data point $\ln k_{e x}{ }^{(i)}$ was set to $\ln k_{e x}{ }^{(i+1)}-\ln k_{e x}{ }^{(i-1)}$ to represent the worst reasonable uncertainties. Larger discrepancies in Inkex ${ }^{(i)}$ would require to alter the order of the simulated spectra of Fig. 3 with respect to the experimental ones. A linear fit (dashed line) yields an activation energy $E_{a}=36.0 \pm 1.5 \mathrm{kJmol}^{-1}$ and pre-exponential factor $A=8.4 \times 10^{15} \mathrm{~s}^{-1}$. 
Cross-term splittings due to the orientational inequivalence of proton magnetic shielding tensors: Do water molecules trapped in crystals hop or tunnel?
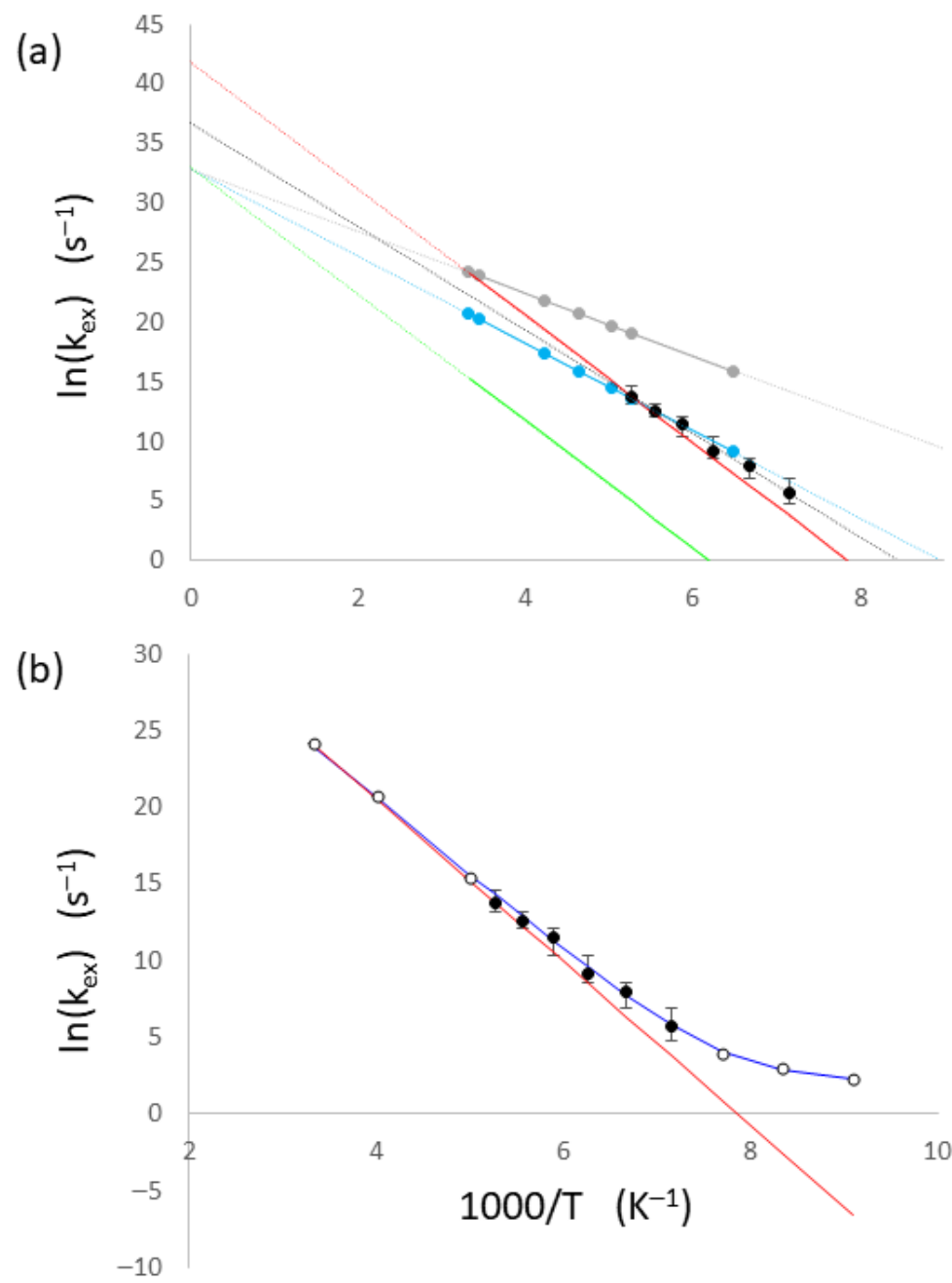

Figure 5 (a) Temperature dependence of the exchange rates $\ln \left(k_{e x}\right)$ estimated by comparison of the experiments and simulations of Fig. 3 for $140<\mathrm{T}<190 \mathrm{~K}$ (black dots with error bars). A linear fit is indicated by a black dashed line and yields $E_{a}=36.0 \pm 1.5 \mathrm{kJmol}^{-1}$ and $A=8.4 \times 10^{15}(\ln A=36.7 \pm 1.0)$. Analogous data derived from the ${ }^{2} \mathrm{H}$ NMR study of Long et al. ${ }^{19}$ are shown by cyan dots $\left(E_{a}=30.4 \mathrm{kJmol}^{-1}\right.$ and $\left.A=1.9 \times 10^{14}\right)$. In grey, these latter data points are adapted for ${ }^{1} \mathrm{H}$ by assuming the usual kinetic isotope effect $\left(E_{a}\left({ }^{1} \mathrm{H}\right)=E_{a}\left({ }^{2} \mathrm{H}\right) / 2^{1 / 2}=21.7 \mathrm{kJmol}^{-1}\right.$, keeping the same pre-exponential factor), in agreement with $E_{a}\left({ }^{1} \mathrm{H}\right)==19.2 \mathrm{kJmol}^{-1}$ estimated from the temperature dependence of $\mathrm{T}_{1}\left({ }^{1} \mathrm{H}\right)$ by Silvidi. ${ }^{20}$ The red line indicates an Arrhenius equation with a slope or thermal activation energy $E_{a}=44.2 \mathrm{kJmol}^{-1}$ calculated by planewave pseudopotential DFT methods with an intercept or pre-exponential factor $A=$ $1.4 \times 10^{18}$ chosen so as to match the $k_{e x}$ of the ${ }^{1} \mathrm{H}$ case (grey data point) at high temperature ( $\mathrm{T}=300 \mathrm{~K}$, left-most grey data point). The green line has the same slope as the red one, with a pre-exponential factor equal to that of Long et al. ${ }^{19}$ (b) Plot of the equation 3 (blue line) for the Bell-Limbach model where contributions of incoherent quantum tunneling are taken into account assuming our DFT parameters, and superimposed onto our experimental data points from (a) where six additional points have been added to indicate the temperatures for which simulated spectra are shown in Fig. 3. Data points are shown as empty dots without error bars in regions where the FWHH does not vary significantly with the temperature. 
The cyan data points represent the ${ }^{2} \mathrm{H}$ exchange rates as measured by Long et al. which lead to an activation energy $E_{a}=30.4 \mathrm{kJmol}^{-1}$ and pre-exponential factor $A=1.5 \times 10^{14} \mathrm{~s}^{-1} .{ }^{19} \mathrm{In}$ grey, these latter data points are adapted for the ${ }^{1} \mathrm{H}$ case by assuming the usual kinetic isotope effect $\left(E_{a}\left({ }^{1} \mathrm{H}\right)=E_{a}\left({ }^{2} \mathrm{H}\right) / 2^{1 / 2}=21.7 \mathrm{kJmol}^{-1}\right.$, keeping the same pre-exponential factor $),{ }^{19}$ in agreement with $E_{a}\left({ }^{1} \mathrm{H}\right)=19.2 \mathrm{kJmol}^{-1}$ estimated by Silvidi from the temperature dependence of $T_{1}\left({ }^{1} \mathrm{H}\right) .{ }^{20}$ Therefore, over the range 140-190 K, our measurements result in exchange rates that are much closer to those found for ${ }^{2} \mathrm{H}$ by Long et al. ${ }^{19}$ than to those estimated for ${ }^{1} \mathrm{H}$ by Silvidi. ${ }^{20}$ Furthermore, within our experimental errors, the activation energy measured in our ${ }^{1} \mathrm{H}$ study is comparable if not larger than that found for ${ }^{2} \mathrm{H}$. In order to rationalize these findings, we investigated the activation energy associated with a classical thermally activated exchange process by means of a series of single-point energy (SPE) calculations performed with planewave pseudopotential DFT methods on the periodic lattice. A single water molecule within one geometry-optimized unit cell was rotated about the $\mathrm{H}-\mathrm{O}-\mathrm{H}$ bisector in steps of $18^{\circ}$ from 0 to $180^{\circ}$. SPE calculations were performed on the resulting geometries. The corresponding energy profile, shown in Supporting Information (Fig. S2), yields an activation energy $E_{a}=44.2$ $\mathrm{kJmol}^{-1}$. The red line in Figure $5(\mathrm{a})$ indicates the slope obtained with this calculated value when the pre-exponential factor $A=1.4 \times 10^{18} \mathrm{~s}^{-1}$ is chosen to match the experimental rate $k_{e x}=3 \times$ $10^{10} \mathrm{~s}^{-1}$ for ${ }^{1} \mathrm{H}$ at $T=300 \mathrm{~K}$. In contrast, the green line indicates an Arrhenius plot obtained for $E_{a}$ $=44.2 \mathrm{kJmol}^{-1}$ estimated by DFT methods in this study but keeping the same pre-exponential factor $A=1.9 \times 10^{14}$ found for ${ }^{2} \mathrm{H}$ by Long et al. ${ }^{19} \mathrm{In}$ this latter case the agreement with the experimental data points is evidently very poor. Both red and green lines in Fig. 5(a) indicate that both activation energies and pre-exponential factors determined experimentally in this study and those reported in the literature are smaller than those calculated by DFT methods.

In order to explain these discrepancies, we considered effects of quantum tunneling, as described by Bell. ${ }^{22}$ More generally, quantum tunneling phenomena have been investigated in a variety of chemical contexts, with particular attention to their role in kinetic isotope effects. ${ }^{30-38}$ Within this framework, the probability of a quantum object such as a proton with an energy smaller than the potential barrier to appear on the other side is taken into account in the evaluation of the apparent rate constant of the process. This probability is greater for particles 
with smaller masses and for narrower barriers. Bell's model has been extensively utilized by Limbach and collaborators for a variety of systems. ${ }^{23,32}$ The blue line in Fig. 5 (a) shows the exchange rates predicted by the Bell-Limbach model in the case at hand when the height of the barrier is calculated by DFT. The same experimental data points of (a) are represented again in (b) by black dots, but six further six further points (open circles) have been added, corresponding to temperatures for which the spectra have been simulated in Fig. 3. These latter points do not show any error bars as they are related to motional regimes where the linewidth is not significantly influenced by changes in temperature, as shown in Fig. 4. The agreement between the Bell-Limbach model and our experimental data points is qualitatively satisfying over the entire temperature range. Figure 5 (b) shows that a model that includes a thermally activated process at high temperatures and contributions of incoherent quantum tunneling at low temperatures describes our experimental data in the 140-190 $\mathrm{K}$ range in a satisfactory manner and agrees with our DFT calculations of the classical barrier height.

For two interleaved spinning sideband manifolds to be observed in low-temperature spectra, as shown in Fig 2(b), two necessary conditions must be fulfilled: (i) the two protons must have two orientationally inequivalent (non-parallel) CSA tensors, (ii) they must have a mutual dipole-dipole coupling. The proof that these two conditions must both be fulfilled is given in the Supporting Information. Figure S3(a) shows a simulated MAS spectrum of a twospin system without exchange, like Fig 2(b). Figure S3(b) shows an analogous simulation where the orientational inequivalence of the two shielding tensors was removed by assuming that the two shielding tensors were collinear (and orthogonal to the dipolar tensor). This assumption leads to a single spinning sideband manifold, with an overall spread that reflects the strength of the intramolecular dipolar-dipole coupling, like the width of the Pake pattern of a static nonspinning powder. This proves that the cross-term splitting between the two sideband manifolds observed at low temperatures requires orientational inequivalence. Furthermore, Fig. S3(c) shows a simulated spectrum where the dipolar interaction was removed. One observes again a single spinning sideband pattern, albeit with a much narrower range because the CSA at 18.8 T is much smaller than the dipolar interaction. Clearly, the cross-term splitting between the two 
Cross-term splittings due to the orientational inequivalence of proton magnetic shielding tensors: Do water molecules trapped in crystals hop or tunnel?

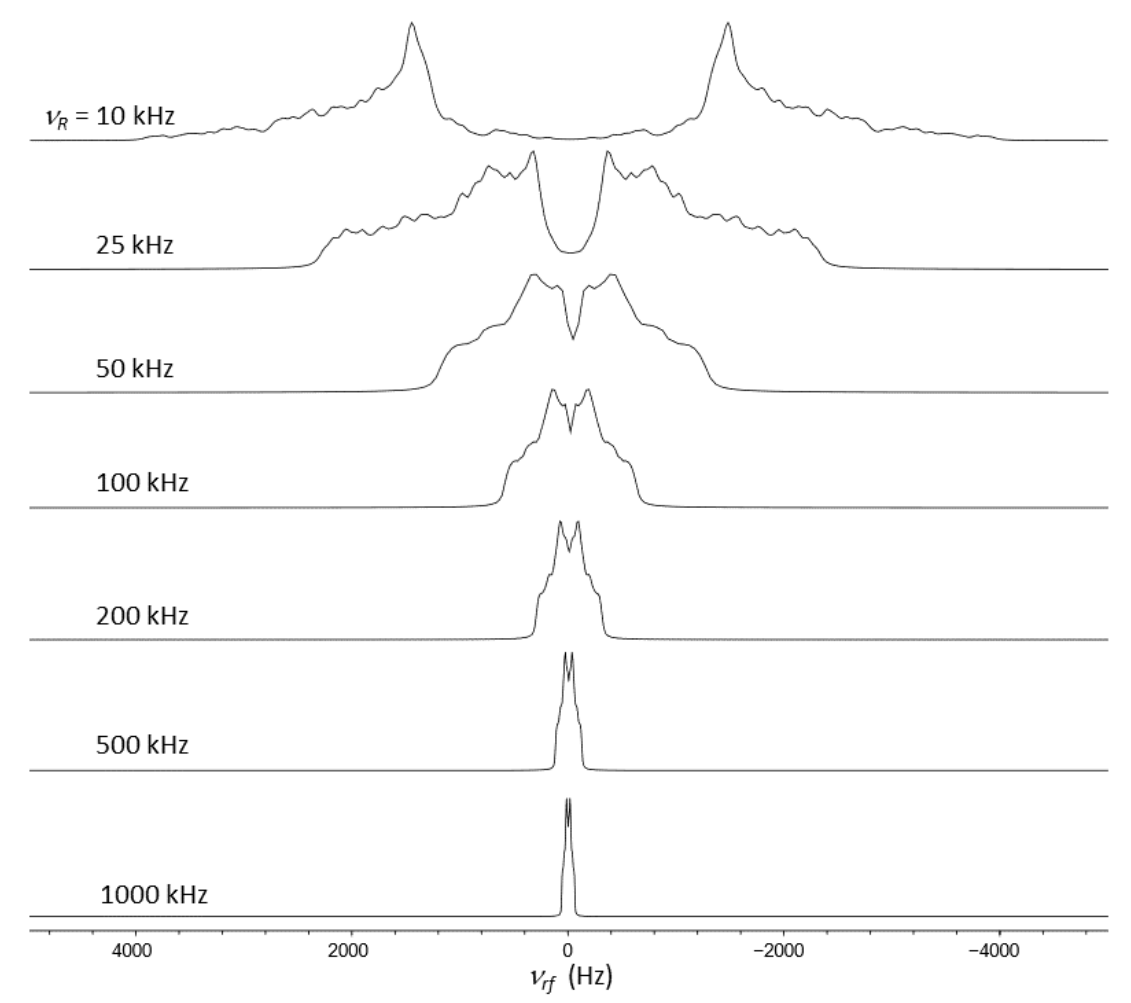

Figure $6 \quad$ Simulated ${ }^{1} \mathrm{H}$ MAS spectra analogous to Fig. 3 for different spinning rates $v_{R}$ but without inclusion of chemical exchange. A rotor-synchronized detection was assumed in all cases. Powder averaging involved $8 \times 320$ crystallites.

interleaved sideband manifolds observed in proton MAS spectra at low temperatures only occur when both above conditions are fulfilled simultaneously.

The unusual splitting observed at low temperatures results from cross terms between the predominant intramolecular homonuclear dipolar coupling and the two shielding tensors provided these are orientationally inequivalent. Similar cross terms have been extensively studied in solution-state NMR, where they lead to cross-correlated relaxation effects. ${ }^{39}$ In solids, the resulting cross terms lead to fourth-rank effects that cannot be eliminated by magic angle spinning. ${ }^{24,25}$ This is shown in Figure 6, where the effect of the spinning rate on the cross-term splittings observed in this study is investigated by numerical simulations.

Experimental attempts at either saturating or inverting one of the two spinning sideband manifolds observed at low temperature by means of a train of rotor-synchronized DANTE 
Cross-term splittings due to the orientational inequivalence of proton magnetic shielding tensors: Do water molecules trapped in crystals hop or tunnel?

pulses $^{40-43}$ were unsuccessful, since the other non-irradiated manifold was also affected (data not shown). This can be understood with the help of the simulations shown in Supporting Information (Fig. S4 and S5), where either single-spin or single-transition operators were detected in both static and spinning cases.

\section{Conclusions}

The analysis of hitherto unreported cross-term splittings between two manifolds of spinning sidebands in proton MAS spectra has allowed us to investigate the dynamics of protons of water molecules confined in crystals of barium chlorate monohydrate over a wide temperature range $110 \leq T \leq 300 \mathrm{~K}$. Numerical simulations that include proton exchange between two sites with non-equivalent (i.e., non-collinear) anisotropic chemical shift tensors reproduce the experimental spectral features remarkably well. The Arrhenius plot was compared with ${ }^{2} \mathrm{H}$ and ${ }^{1} \mathrm{H}$ NMR data of the same sample presented in the literature. The activation energy for the two-fold hop about the $\mathrm{H}-\mathrm{O}-\mathrm{H}$ bisector was evaluated by means of planewave-pseudopotential DFT methods. The Bell-Limbach model that includes contributions due to both a thermally activated processes and incoherent quantum tunneling is consistent with the experimental data over the entire explored temperature range. The non-Lorentzian lineshapes observed at low temperatures stem from cross terms due to interference between CSA and homonuclear dipolar interactions. Selective irradiation experiments prove that the two interleaved spinning sideband manifolds cannot be disentangled.

\section{Experimental and computational details}

The sample was purchased from Sigma-Aldrich and kept overnight in an oven at $70^{\circ} \mathrm{C}$ prior to use in order to minimize adsorption water. All proton MAS spectra were recorded the Ecole Normale Supérieure on a wide-bore Bruker 800 spectrometer (18.8 T) with an Avance-III console, using $3.2 \mathrm{~mm} \mathrm{ZrO}_{2}$ rotors spinning at $10 \mathrm{kHz}$ in a triple-resonance low-temperature MAS probe designed for DNP experiments, but without microwave irradiation. The $r f$-field amplitude of the proton pulses was $v_{1}=125 \mathrm{kHz}$, corresponding to a pulse length $\tau_{p}=2 \mu \mathrm{s}$ for a $90^{\circ}$ nutation angle. All spectra were acquired with rotor-synchronized solid echoes $\left(90^{\circ}-\tau-90^{\circ}\right.$ - $\tau$ - detection.) Exorcycle ${ }^{44}$ was applied to the second $90^{\circ}$ pulse. DFT calculations were 
Cross-term splittings due to the orientational inequivalence of proton magnetic shielding tensors: Do water molecules trapped in crystals hop or tunnel?

conducted with Quantum ESPRESSO 6.3. ${ }^{45,46}$ Geometry optimization was performed on the known crystal structure ${ }^{26}$ utilizing the Perdew-Burke-Ernzerhof (PBE) functional, ${ }^{47}$ a cut-off energy of $80 \mathrm{Ry}, 4 \times 4 \times 4$ k-point grid, ultrasoft ${ }^{48}$ pseudopotentials for $\mathrm{Ba}$ and $\mathrm{Cl}$ atoms and norm-conserving ${ }^{49}$ pseudopotentials for $\mathrm{O}$ and $\mathrm{H}$ atoms. After convergence, the geometry was characterized by total forces acting on all atoms smaller than 0.0038 Ry/Bohr. Cartesian coordinates and cell parameters are given in the Supporting Information. The optimized structure was subsequently used for a series of single-point energy (SPE) calculations to estimate the potential barrier of the $180^{\circ}$ flip of one water molecule about the $\mathrm{H}-\mathrm{O}-\mathrm{H}$ bisector. NMR calculations were performed on the crystal structure optimized with a cut-off energy of 100 Ry, $4 \times 4 \times 4$ k-point grid and norm-conserving pseudopotentials for all atoms. The gaugeincluding projector augmented wave (GIPAW) algorithm was employed to reconstruct allelectron wave functions in the presence of a magnetic field. ${ }^{50,51}$ The graphical tool MagresView ${ }^{52}$ was utilized to extract the sets of Euler angles relating the orientations of the relevant tensorial interactions when expressed in a common crystal frame by a DFT calculation of NMR parameters.

The numerical simulations in Fig. 3 of MAS spectra obtained by Fourier transformation of solid echoes with inclusion of chemical exchange were carried out by means of the master equation, as applied to solids by Duer and Levitt: ${ }^{53,54}$

$$
\boldsymbol{\rho}(t)=\exp \left\{\left(-i L_{c o h}+L_{e x}\right) \Delta t\right\} \boldsymbol{\rho}(0),
$$

where $L_{\text {coh }}$ is the commutation superoperator describing coherent spin dynamics due to the anisotropic chemical shift and dipolar Hamiltonians, i.e., all interactions relevant in our case as far as the water molecules can be considered to be isolated. Since these interactions act on an initial state $\boldsymbol{\rho}(0)$ comprising only $I_{x}$ and $S_{x}$, it is sufficient to consider an $8 \times 8$ single-quantum subspace spanned by the basis $B=\left\{I_{x}, I_{y}, S_{x}, S_{y}, 2 I_{x} S_{z}, 2 I_{y} S_{z}, 2 I_{z} S_{x}, 2 I_{z} S_{y}\right\}$. In this basis, the corresponding superoperator $L_{e x}$ describing incoherent dynamics due to chemical exchange between the $I$ and $S$ spins is: 

water molecules trapped in crystals hop or tunnel?

$$
L_{e x}=\left(\begin{array}{cccccccc}
k_{e x} & 0 & -k_{e x} & 0 & 0 & 0 & 0 & 0 \\
0 & k_{e x} & 0 & -k_{e x} & 0 & 0 & 0 & 0 \\
-k_{e x} & 0 & k_{e x} & 0 & 0 & 0 & 0 & 0 \\
0 & -k_{e x} & 0 & k_{e x} & 0 & 0 & 0 & 0 \\
0 & 0 & 0 & 0 & k_{e x} & 0 & -k_{e x} & 0 \\
0 & 0 & 0 & 0 & 0 & k_{e x} & 0 & -k_{e x} \\
0 & 0 & 0 & 0 & -k_{e x} & 0 & k_{e x} & 0 \\
0 & 0 & 0 & 0 & 0 & -k_{e x} & 0 & k_{e x}
\end{array}\right) .
$$

Numerical simulations without exchange processes were conducted in Hilbert space by means of the Simpson program. ${ }^{55}$

The apparent exchange rate $k_{T}$ that includes contributions from both thermal activation and quantum tunneling can be expressed by: ${ }^{23}$

$$
k_{T}=\frac{A}{R T} \int_{0}^{\infty} \exp \left(\frac{-W}{R T}\right)\left[1+\exp \left(\frac{V_{0}-W}{h v_{T}}\right)\right]^{-1} d W
$$

where $A$ is the classical Arrhenius-type pre-exponential factor, $V_{0}$ the barrier height, and the tunneling frequency $v_{T}$ is given by: ${ }^{23}$

$$
v_{T}=\frac{1}{\pi a} \sqrt{\frac{E_{0}}{2 m}},
$$

where $m$ is the total mass of the two tunneling protons and $a$ the barrier width at height $E_{0}$ $<V_{0}$. The integral in Eq. (1) was evaluated numerically over the interval $0 \leq W \leq 20 V_{0}$. The

parameters utilized in Fig. 5(b) are $A=9.0 \times 10^{17} \mathrm{~s}^{-1}, E_{0}=18.6 \mathrm{kJmol}^{-1}, a=(\pi / 2) \times 1.61 \AA$ and $m=2 \times 1.673 \times 10^{-27} \mathrm{~kg}$, corresponding to 2 proton masses traveling through a barrier with a thickness given by the semicircle spanned by a $180^{\circ}$ rotation of an $\mathrm{H}_{2} \mathrm{O}$ molecule about the $\mathrm{H}-\mathrm{O}-\mathrm{H}$ bisector as taken from the DFT-optimized crystal structure.

\section{Acknowledgements}

The authors thank Hans Limbach, Maurice Goldman, Bob Griffin, Jean Jeener and Malcolm Levitt for constructive comments and Nicolas Birlirakis and Mathieu Baudin for assistance with experiments. This work was supported by the CNRS, the European Research Council (ERC, 
Cross-term splittings due to the orientational inequivalence of proton magnetic shielding tensors: Do water molecules trapped in crystals hop or tunnel?

contract 'dilute para-water', grant agreement number 339754), and the French "Equipements d'Excellence" Paris-en-Resonance, contract ANR-10-EQPX-09.

\section{References}

1. Ball, P. Water - an enduring mystery. Nature 452, 291-292 (2008).

2. Finney, J. L. Water? What's so special about it? Phil. Trans. R. Soc. B 359, 1145 (2004).

3. Angell, C. A. Insights into Phases of liquid water from study of its unusual glass-forming properties. Science 319, 582 (2008).

4. Israelachvili, J. Wennerström, H. Role of hydration and water structure in biological and colloidal interactions. Nature 379, 219 (1996).

5. Algara-Siller, G. et al. Square ice in graphene nanocapillaries. Nature 519, 443 (2015).

6. Koga, K., Gao, G. T., Tanaka, H. \& Zeng, X. C. Formation of ordered ice nanotubes inside carbon nanotubes. Nature 412, 802 (2001).

7. Kolesnikov, A. I. et al. Anomalously soft dynamics of water in a nanotube: a revelation of nanoscale confinement. Phys. Rev. Lett. 93, 035503 (2004).

8. Hernández de la Peña, L. \& Kusalik, P. G. Temperature dependence of quantum effects in liquid Water. J. Am. Chem. Soc. 127 (14), 5246-5251 (2005).

9. Paesani, F., luchi, S. \& Voth, G. A. Quantum effects in liquid water from an ab initio-based polarizable force field. J. Chem. Phys. 127, 074506 (2007).

10. Drechsel-Grau, C. \& Marx, D. Collective proton transfer in ordinary ice: local environments, temperature dependence and deuteration effects. Phys. Chem. Chem. Phys. 19, 2623-2635 (2017).

11. Gorshunov, B. P. et al. Quantum behavior of water molecules confined to nanocavities in gemstones. J. Phys. Chem. Lett. 4(12), 2015-2020 (2013).

12. Kolesnikov, A. I. et al. Quantum tunneling of water in beryl: a new state of the water molecule. Phys. Rev. Lett. 116, 167802 (2016).

13. Richardson, J. O. et al. Concerted hydrogen-bond breaking by quantum tunneling in the water hexamer prism. Science 351, 6279, 1310-1313 (2016).

14. Mammoli, D. et al. Challenges in preparing, preserving and detecting para-water in bulk: overcoming proton exchange and other hurdles. Phys. Chem. Chem. Phys. 17, 26819-26827 (2015).

15. Meier, B. et al. Spin-isomer conversion of water at room temperature and quantum-rotor-induced nuclear polarization in the water-endofullerene $\mathrm{H}_{2} \mathrm{O} @ \mathrm{C}_{60}$. Phys. Rev. Lett. 120, 266001 (2018).

16. Eisendrath, H., Stone, W. \& Jeener, J. NMR of protons in gypsum. I. Experimental proof of the existence of four thermodynamic invariants. Phys. Rev. B 17, 1, 47-53 (1978).

17. Eisendrath, H. \& Jeener, J. NMR of protons in gypsum. II. Independent observation of molecular reorientations and molecular diffusion. Phys. Rev. B 17, 1, 54-60 (1978).

18. Pake, G. E. Nuclear resonance absorption in hydrated crystals: fine structure of the proton line. J. Chem. Phys. 16, 327-336 (1948).

19. Long, J. R., Ebelhäuser, R. \& Griffin, R. G. ${ }^{2} \mathrm{H}$ NMR Line Shapes and Spin-Lattice Relaxation in $\mathrm{Ba}\left(\mathrm{ClO}_{3}\right)_{2} \cdot{ }^{2} \mathrm{H}_{2} \mathrm{O}$. J. Phys. Chem. A 101, 988-994 (1997). 
Cross-term splittings due to the orientational inequivalence of proton magnetic shielding tensors: Do water molecules trapped in crystals hop or tunnel?

20. Silvidi, A. A. Protic spin-lattice relaxation in some hydrated barium salt crystals. J. Chem. Phys. 45, 38923895 (1966).

21. Keeler, E. G., Michaelis, V. K. \& Griffin, R. G. ${ }^{17} \mathrm{O}$ NMR investigation of water structure and dynamics. J. Phys. Chem. B 120, 7851-7858 (2016).

22. Bell, R. P. The Tunnel Effect in Chemistry (Chapman and Hall, London, 1980).

23. Buntkowsky, G. \& Limbach, H.-H. H-solid state NMR studies of tunneling phenomena and isotope effects in transition metal dihydrides. J. Low Temp. Phys. 143, 55-114 (2006).

24. Maricq, M. M. \& Waugh, J. S. NMR in rotating solid. J. Chem. Phys. 70, 3300-3316 (1979).

25. Ashbrook, S. E., McManus, J., Thrippleton, M. J. \& Wimperis, S. Second-order cross-term interactions in high-resolution MAS NMR of quadrupolar nuclei. Prog. Nucl. Magn. Reson. Spectrosc. 55, 160-181 (2009).

26. Sikka, S. K., Momin, S. N., Rajagopal, H. \& Chidambaram, R. Neutron-diffraction refinement of the crystal structure of barium chlorate monohydrate $\mathrm{Ba}\left(\mathrm{ClO}_{3}\right)_{2} \cdot \mathrm{H}_{2} \mathrm{O}$. J. Chem. Phys. 48, 1883 (1968).

27. Carnevale, D., Ashbrook, S. E. \& Bodenhausen, G. Solid-state NMR measurements and DFT calculations of the magnetic shielding tensors of protons of water trapped in barium chlorate monohydrate. RSC Adv. 4, 56248-56258 (2014).

28. Carnevale, D., Chinthalapalli, S. \& Bodenhausen, G. Exciting wide NMR spectra of static solid samples with weak radiofrequency fields. Z. Phys. Chem. 231, 3, 527-543 (2017).

29. Vold, R. R. \& Vold, R. L. Deuterium relaxation in molecular solids. Adv. Magn. Opt. Reson. 16, 85-171 (1991).

30. Shiner Jr. V. J. \& Smith, M. L. The Arrhenius parameters of the deuterium isotope rate effect in a basepromoted elimination reaction ${ }^{1}$ : evidence for proton tunnelling. J. Am. Chem. Soc. 83, 593-598 (1961).

31. Cha, Y., Murray, C. \& Klinman, J. Hydrogen tunneling in enzyme reactions. Science 243, 1325-30 (1989).

32. Limbach, H.-H., Hennig, J., Gerritzen, D. \& Rumpel, H. Primary kinetic HH/HD/DH/DD isotope effects and proton tunnelling in double proton-transfer reactions. Faraday Discuss. Chem. Soc. 74, 229-243 (1982).

33. Limbach, H.-H. et al. NMR and INS Line Shapes of Transition Metal Hydrides in the Presence of Coherent and Incoherent Dihydrogen Exchange. J. Am. Chem. Soc. 120, 7929-7943 (1998).

34. Wehrmann, F., Fong, T. P., Morris, R. H., Limbach, H.-H. \& Buntkowsky, G. Coherent D2 rotational tunneling and incoherent D2 dynamics in a solid non-classical RuD2 complex studied by $2 \mathrm{H}$ solid state NMR spectroscopy. Phys. Chem. Chem. Phys. 1, 4033-4041 (1999).

35. Limbach, H.-H., Miguel Lopez, J. \& Kohen, A. Arrhenius curves of hydrogen transfers: tunnel effects, isotope effects and effects of pre-equilibria. Philos. Trans. R. Soc. Lond. B Biol. Sci. 361, 1399-1415 (2006).

36. Meisner, J. \& Kästner, J. Atom tunneling in chemistry. Angew. Chem. Int. Ed. 55, 5400- 5413 (2016).

37. Horsewill, A. J. Quantum tunnelling aspects of methyl group rotation studied by NMR. Prog. Nucl. Magn. Reson. Spectrosc. 35, 359-389 (1999).

38. Pohl, R. et al. Proton transfer in guanine-cytosine base pair analogues studied by NMR spectroscopy and PIMD simulations. Faraday Discuss. 212, 331-344 (2018).

39. Kumar, A., Grace, R. C. R. \& Madhu, P. K. Cross-correlations in NMR. Prog. Nucl. Magn. Reson. Spectrosc. 37, 191-319 (2000).

40. Caravatti, P., Bodenhausen. G. \& Ernst, R. R. Selective pulse experiments in high-resolution solid state NMR. J. Magn. Reson. 55, 88-103 (1983).

41. Carnevale, D. et al. Broadband excitation in solid-state NMR of paramagnetic samples using Delays Alternating with Nutation for Tailored Excitation ('Para-DANTE'). Chem. Phys. Lett. 553, 68-76 (2012). 
Cross-term splittings due to the orientational inequivalence of proton magnetic shielding tensors: Do water molecules trapped in crystals hop or tunnel?

42. Carnevale, D., Perez Linde, A. J., Bauer, G. \& Bodenhausen, G. Solid-state proton NMR of paramagnetic metal complexes: DANTE spin echoes for selective excitation in inhomogeneously broadened lines. Chem. Phys. Lett. 580, 172-178 (2013).

43. Lu, X. et al. Broadband excitation in solid-state NMR using interleaved DANTE pulse trains with $\mathrm{N}$ pulses per rotor period. J. Magn. Reson. 236, 105-116 (2013).

44. Bodenhausen, G., Freeman, R. \& Turner, D. L. Suppression of artifacts in two-dimensional J spectroscopy. J. Magn. Reson. 27, 511-514 (1977).

45. Giannozzi, P. et al. QUANTUM ESPRESSO: a modular and open-source software project for quantum simulations of materials. J. Phys.: Condens. Matter 21, 395502 (2009).

46. Giannozzi, P. et al. Advanced capabilities for materials modelling with Quantum ESPRESSO. J. Phys.: Condens. Matter 29, 465901 (2017).

47. Perdew, J. P., Burke, K. \& Ernzerhof, M. Generalized Gradient Approximation Made Simple. Phys. Rev. Lett. 77, 18, 3865-3868 (1996).

48. Vanderbilt, D. Soft self-consistent pseudopotentials in a generalized eigenvalue formalism. Phys. Rev. B 41, 7892-7895 (1990).

49. Hamann, D. R., Schlüter, M. \& Chiang, C. Norm-Conserving Pseudopotentials. Phys. Rev. Lett. 43, 14941497, (1979).

50. Pickard, C. J. \& Mauri, F. All-electron magnetic response with pseudopotentials: NMR chemical shifts. Phys. Rev. B 63, 245101 (2001).

51. Yates, J. R., Pickard, C. J. \& Mauri, F. Calculation of NMR chemical shifts for extended systems using ultrasoft pseudopotentials. Phys. Rev. B 76, 024401 (2007).

52. Sturniolo, S. et al. Visualization and processing of computed solid-state NMR parameters: MagresView and MagresPython. Solid State Nucl. Magn. Reson. 78 64-70 (2016).

53. Ernst, R. R., Bodenhausen, G. \& Wokaun, A. Principles of Nuclear Magnetic Resonance in One and Two Dimensions (Clarendon Press, Oxford 1987).

54. Duer, M. J. \& Levitt, M. H. Time-domain calculation of chemical exchange effects in the NMR spectra of rotating solids. Solid State Nucl. Magn. Reson. 1, 211-215 (1992).

55. Bak, M., Rasmussen, J. T. \& Nielsen, N. C. SIMPSON: A General Simulation Program for Solid-State NMR Spectroscopy. J. Magn. Reson. 147, 296-330 (2000). 